\title{
Optimal Bit and Power Loading for Amplify-and-Forward Cooperative OFDM Systems
}

\author{
Osama Amin, Student Member, IEEE, and Murat Uysal, Senior Member, IEEE
}

\begin{abstract}
In this paper, we investigate bit and power allocation strategies for an orthogonal frequency division multiplexing (OFDM) cooperative network over frequency-selective fading channels. We assume amplify-and-forward relaying and consider the bit error rate (BER) performance as our performance measure. Aiming to optimize the BER under total power constraint and for a given average data rate, we propose three adaptive algorithms; optimal power loading (OPL), optimal bit loading (OBL), and optimal joint bit and power loading (OBPL). Our Monte Carlo simulation results demonstrate performance gains through adaptive bit and power loading over conventional non-adaptive systems as well as currently available adaptive cooperative scheme in the literature. The impact of practical issues on the performance of proposed adaptive schemes such as imperfect channel estimation and limited feedback is further discussed.
\end{abstract}

Index Terms-OFDM, power allocation, bit allocation, amplify-and-forward relaying, cooperative network.

\section{INTRODUCTION}

$\mathbf{E}$ XPLOITING the broadcasting nature of wireless transmission, cooperative diversity forms a virtual antenna array among the single-antenna nodes and extracts the spatial diversity advantages in a distributed setting [1], [2]. Most of the existing work in the area of cooperative diversity is based on the assumption of open-loop implementation where the destination node and (possibly) relay nodes have channel knowledge via channel estimation, while transmitting nodes have no knowledge of the channel. Such open-loop designs are favourable in time-varying channels where feedback of channel estimates becomes problematic. However, particularly for fixed wireless access applications, reliable feedback is possible and available channel state information (CSI) at the transmitting nodes can be used to design adaptive transmission schemes for performance improvement.

Adaptive transmission has been extensively studied in the context of both single-carrier and multi-carrier point-to-point links, see e.g., [3]-[8] and the references therein. Adaptive transmission has been recently applied to cooperative diversity

Manuscript received August 14, 2009; revised April 14, 2010, August 30, 2010, and October 7, 2010; accepted October 7, 2010. The associate editor coordinating the review of this paper and approving it for publication was S. Bhashyam.

This paper was presented in part at the 11th Canadian Workshop on Information Theory (CWIT09), Ottawa, Ontario, Canada, May 2009.

O. Amin is with the Department of Electrical and Computer Engineering, University of Waterloo, Waterloo, ON, N2L3G1, Canada (e-mail: oamin@uwaterloo.ca). The work of O. Amin is supported by the Egypian Higher Education Ministry.

M. Uysal is with the Faculty of Engineering, Özyeğin University, 34662, Istanbul, Turkey (e-mail: murat.uysal@ozyegin.edu.tr).

Digital Object Identifier 10.1109/TWC.2011.011111.091233 systems. In [9], Ahmed et al. assume a single relay system with amplify-and-forward (AF) relaying over frequencyflat Rayleigh fading channels. They propose power control schemes to minimize the outage probability for fixed rate transmission. In [10], Ahmed and Aazhang design rate and power control algorithms for decode-and-forward (DF) relaying. A theoretical study for the achievable rates in adaptive DF and compress-and-forward relaying is further introduced in [11]. In [12], [13] Hammerstom and Wittneben consider frequency-selective channels and propose optimum power control to maximize the instantaneous rate for an uncoded two-hop OFDM scheme. Unlike [12], [13] which adopts AF relaying, Ying et al. [14] consider DF relaying and investigate adaptive OFDM cooperative systems with orthogonal AF cooperation protocol [2] (also known as receive diversity (RD) protocol [15]). In [16], Gui and Cimini also consider $\mathrm{RD}$ protocol and present bit and power loading algorithms for OFDM DF cooperative systems with sub-carrier selection to minimize the total transmission power under a fixed rate assumption. In another work which builds on $\mathrm{RD}$ protocol [17], [18], Ma et al. propose a sub-optimal bit and power loading algorithm for OFDM cooperative system to maximize the throughput under individual power constraints and a target link error rate assuming AF and selective DF relaying. In [19], the same authors study bit and power loading algorithms to minimize the transmit power consumption for $\mathrm{AF}$ and selective DF modes at a target throughput. In [20], Hajiaghayi et al. address power loading for an OFDM AF system with RD protocol. They formulate two problems; one aims to maximize the system capacity and the other aims to minimize the bit error rate (BER).

In most of the existing literature on adaptive OFDM [12][14], [16]-[20], objective function is chosen as either to maximize the throughput or minimize the power consumption. An exception is [20] which addresses power loading for adaptive OFDM to minimize BER performance in a single-relay scenario. However, their solution is sub-optimal since they work under the high signal-to-noise ratio (SNR) assumption and consider identical subcarrier power at source and relay nodes. In this paper, we aim to optimize BER performance and propose three adaptive bit or/and power loading algorithms for single-relay OFDM system with AF relaying and RD protocol. The first algorithm computes the optimal source and relay power loading coefficients under total power constraint and fixed subcarriers' rate. The second algorithm computes the optimal bit loading coefficients under fixed average transmission rate and equal power loading. The third algorithm computes the joint optimal power and bit loading coefficients under total power constraint and fixed average transmission 
rate. Through Monte-Carlo simulations, we demonstrate that our proposed schemes achieve full diversity and outperform conventional schemes with equal power loading as well as precoded systems. We further investigate the effect of practical considerations such as imperfect CSI and quantization on the performance.

The rest of the paper is organized as follows: The signal model for cooperative OFDM system under consideration is described in Section II. Optimization problem to minimize BER is first formulated in Section III and then corresponding power and/or bit loading algorithms are presented. The simulated performance of proposed adaptive schemes is presented in Section IV. And finally Section V summarizes and concludes the paper.

Notation: Bold upper-case letters denote matrices and bold lower-case letters denote vectors. $\mathbb{E}$ and $|$.$| denote respectively$ the expectation and the absolute value. $(.)^{T}$ and $(.)^{H}$ denotes transpose and conjugate transpose (i.e., Hermitian) operations, respectively. $Q($.$) represents the Gaussian Q$ function. $\mathbf{Q}$ represents the $N \times N$ fast Fourier transform (FFT) matrix, i.e. $\mathbf{Q}(p, q)=(1 / \sqrt{N}) \exp (-j 2 \pi(p-1)(q-1) / N), p=$ $0,1, \ldots, N-1, q=0,1, \ldots, N-1$.

\section{TRANSMISSION MODEL}

We consider a cooperative OFDM system with single relay. Source, relay, and destination nodes are equipped with single transmit/receive antennas and operate in halfduplex mode. The nodes are assumed to be located in a two-dimensional plane where $d_{S D}, d_{S R}$ and $d_{R D}$ denote the distances of source-to-destination $(\mathrm{S} \rightarrow \mathrm{D})$, source-to-relay $(\mathrm{S} \rightarrow \mathrm{R})$, and relay-to-destination $(\mathrm{R} \rightarrow \mathrm{D})$ links, respectively (see Fig. 1). In Fig. 1, $\theta$ is the angle between lines representing $\mathrm{S} \rightarrow \mathrm{R}$ and $\mathrm{R} \rightarrow \mathrm{D}$ links.

To explicitly take into account the effect of relay location, we consider both long-term path loss and shortterm frequency-selective Rayleigh fading. The path loss is proportional to $d^{\alpha}$ where $d$ is the distance between nodes and $\alpha$ is the path loss exponent. By normalizing the path loss terms with respect to the direct $\mathrm{S} \rightarrow \mathrm{D}$ link, the so-called geometrical gains can be defined as $G_{S R}=\left(d_{S D} / d_{S R}\right)^{\alpha}$ and $G_{R D}=\left(d_{S D} / d_{R D}\right)^{\alpha}$ [15]. These are related through the cosines law by $G_{S R}^{2 / \alpha}+G_{R D}^{2 / \alpha}-2 G_{S R}^{1 / \alpha} G_{R D}^{1 / \alpha} \cos \theta=G_{S R}^{2 / \alpha} G_{R D}^{2 / \alpha}$. The frequencyselective fading channels are modeled as FIR (finite impulse response) filters with order of $L_{S D}, L_{S R}$ and $L_{R D}$. They are represented by $\mathbf{h}_{S D}=\left[h_{S D}(0), \ldots, h_{S D}\left(L_{S D}\right)\right]^{T}$, $\mathbf{h}_{S R}=\left[h_{S R}(0), \ldots, h_{S R}\left(L_{S R}\right)\right]^{T}$ and $\mathbf{h}_{R D}=$ $\left[h_{R D}(0), \ldots, h_{R D}\left(L_{R D}\right)\right]^{T}$ for $\mathrm{S} \rightarrow \mathrm{D}, \mathrm{S} \rightarrow \mathrm{R}$, and $\mathrm{R} \rightarrow \mathrm{D}$ links, respectively. The entries of $\mathbf{h}_{S D}, \mathbf{h}_{S R}$, and $\mathbf{h}_{R D}$ are assumed to be zero mean, complex Gaussian distribution with their variance equal to $1 /\left(L_{S D}+1\right), 1 /\left(L_{S R}+1\right)$ and $1 /\left(L_{R D}+1\right)$, respectively. The channels are assumed to remain constant over a block of OFDM symbols and change from one block to another independently.

Here, we assume RD cooperation protocol where the source and the relay nodes transmit in orthogonal transmission phases. In the first transmission phase, a bit-stream is fed into serial-to-parallel converter which maps them into modulation

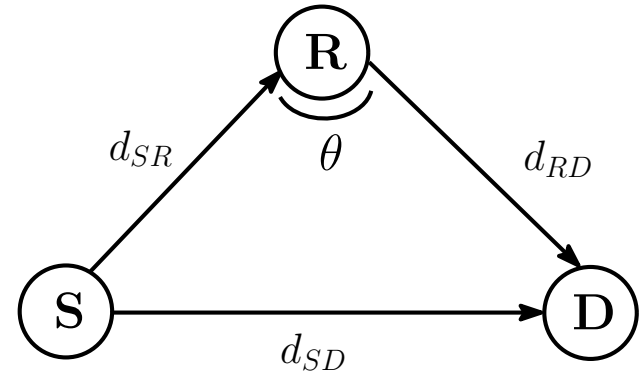

Fig. 1. Cooperative system model.

symbols chosen from either M-PSK or M-QAM constellations. Each $k$ subcarrier symbol carries $b_{k}$ bits based on the employed bit loading algorithm (which will be later introduced). Before passing through inverse FFT (IFFT), the power of each subcarrier symbol is adjusted based on the employed power loading algorithm (which will be later introduced). To prevent inter-block interference, a cyclic prefix $(\mathrm{CP})$ is inserted between OFDM symbols with $L_{C P} \geq \max \left(L_{S R}, L_{R D}, L_{S D}\right)$. Both relay and destination nodes receive the transmitted OFDM symbol. After removing CP and converting the OFDM symbol into parallel subcarrier symbols through FFT, the relay node scales the subcarriers power. Then it feeds the subcarrier symbols to IFFT and adds CP. In the second transmission phase, the relay node forwards the resulting signal to the destination while the source node remains silent. At the destination, both OFDM symbols received during the broadcasting and relaying phases are fed to maximum likelihood (ML) detector after removing $\mathrm{CP}$ and passing through FFT. Block diagrams of source, relay, and destination nodes are provided in Fig. 2.

Let the subcarrier signal for the $k^{\text {th }}$ carrier be denoted as $x(k), k=1,2, \ldots N$ where $N$ is the number of subcarriers. The received signals at the relay and the destination nodes during the broadcasting phase are given by

$$
\begin{gathered}
r_{D_{1}}(k)=\sqrt{E_{k, S}} \exp \left(j \phi_{k, S}\right) D_{S D}(k, k) x(k)+v_{D_{1}}(k) \\
r_{R}(k)=\sqrt{E_{k, S} G_{S R}} \exp \left(j \phi_{k, R}\right) D_{S R}(k, k) x(k)+v_{R}(k)
\end{gathered}
$$

where $E_{k, S}$ and $\phi_{k, S}$ denote, respectively, the adjustable power and phase terms for $k^{\text {th }}$ subcarrier. In the above $v_{D_{1}}(k)$, and $v_{R}(k)$ are the FFT of additive white Gaussian noise (AWGN) terms $n_{D_{1}}(k)$ and $n_{R}(k) . D_{S D}(k, k)$ and $D_{S R}(k, k)$ are the $k^{\text {th }}$ diagonal elements in the diagonal channel matrices $\mathbf{D}_{S D}$ and $\mathbf{D}_{S R}$. They are defined as $\mathbf{D}_{S D}=\operatorname{diag}\left(\mathrm{H}_{\mathrm{SD}}(0), \quad \mathrm{H}_{\mathrm{SD}}(1), \cdots \mathrm{H}_{\mathrm{SD}}(\mathrm{N}-1)\right)$ and $\mathbf{D}_{S R}=\operatorname{diag}\left(\mathrm{H}_{\mathrm{SR}}(0), \quad \mathrm{H}_{\mathrm{SR}}(1), \cdots \mathrm{H}_{\mathrm{SR}}(\mathrm{N}-1)\right)$ where $H_{S D}(k)=\sum_{l=1}^{N-1} h_{S D}(l) \exp (-j 2 \pi l k / N)$ and $H_{S R}(k)=$ $\sum_{l=1}^{N-1} h_{S R}(l) \exp (-j 2 \pi l k / N)$. For the scaling at the relay node, we adopt a slightly modified version of so-called instantaneous power scaling (IPS) [21], [22] which assumes perfect CSI. In IPS, scaling term is given by

$$
\underset{v_{R}(k)}{\mathbb{E}}\left\{\left|r_{R}(k)\right|^{2}\right\}=E_{k, S} G_{S R}\left|D_{S R}(k, k)\right|^{2}+N_{0} .
$$

Here, instead we replace $E_{k, S}$ with the average value $E$. Such a modification does not significantly affect the overall performance, but simplifies the ensuing optimization problem. After scaling the received signal, the relay node amplifies 


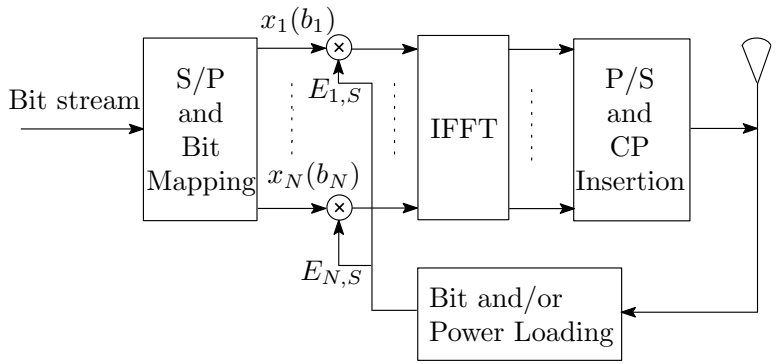

(a) Source

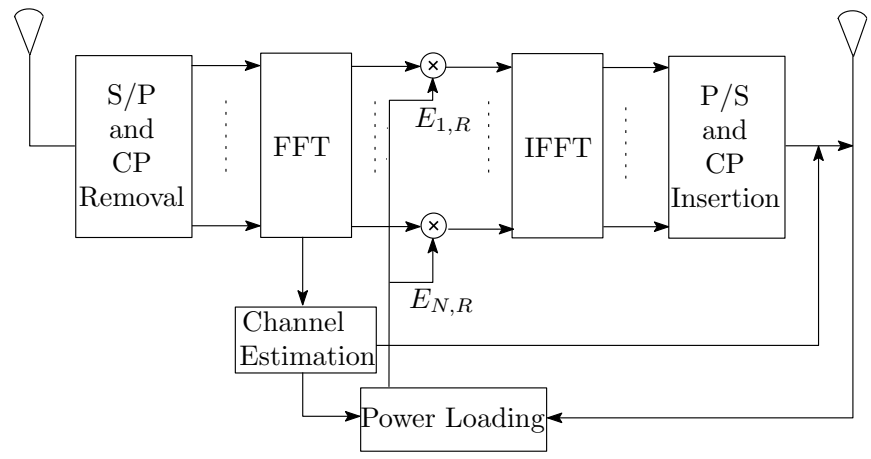

(b) Relay

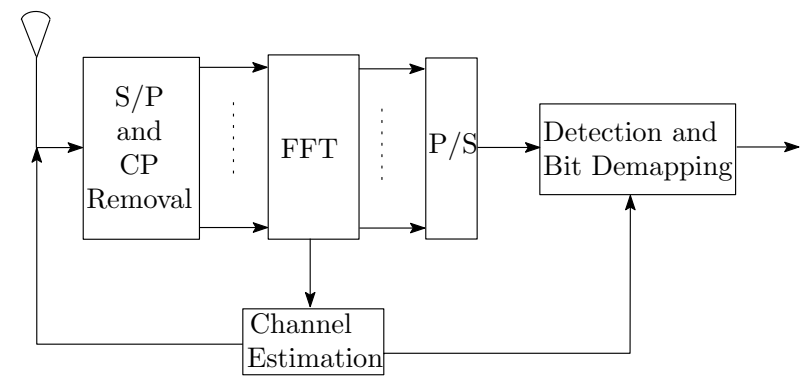

(c) Destination

Fig. 2. Block diagrams of source, relay, and destination nodes.

the $k^{\text {th }}$ subcarrier with power $E_{k, R}$ and adjusts the phase by adding $\phi_{k, R}$. Then it feeds the subcarriers to IFFT and adds $\mathrm{CP}$ before it forwards the resulting signal to destination node.

The destination node removes $\mathrm{CP}$ and converts the received OFDM symbol into subcarrier symbols. The $k^{\text {th }}$ subcarrier signal is given by

$$
\begin{aligned}
r_{D_{2}}(k)= & \frac{\sqrt{G_{S R} G_{R D} E_{k, S} E_{k, R}}}{\sqrt{E G_{S R}\left|D_{S R}(k, k)\right|^{2}+N_{0}}} \exp \left(j\left(\phi_{k, S}+\phi_{k, R}\right)\right) \\
& \times D_{S R}(k, k) D_{R D}(k, k) x(k)+v_{D_{2}}^{\prime}(k)
\end{aligned}
$$

In (4), $v_{D_{2}}^{\prime}(k)$ represents the effective noise term and is given by

$$
v_{D_{2}}^{\prime}(k)=\frac{\sqrt{G_{R D} E_{k, R}} D_{R D}(k, k) \exp \left(j \phi_{k, R}\right)}{\sqrt{E G_{S R}\left|D_{S R}(k, k)\right|^{2}+N_{0}}} v_{R}(k)+v_{D_{2}}(k)
$$

where $v_{D_{2}}(k)$ is the FFT of AWGN term at the destination during the relaying phase. Effective noise is conditionally Gaussian with zero mean and variance of $\varpi=$ $\sqrt{1+E_{k, R} G_{R D}\left|D_{R D}(k, k)\right|^{2} /\left(E G_{S R}\left|D_{S R}(k, k)\right|^{2}+N_{0}\right)}$.
Normalizing (5) with $\varpi$, we have

$\tilde{r}_{D_{2}}(k)=\frac{\sqrt{E_{k, S} E_{k, R}} D_{k, 2} \exp \left(j\left(\phi_{k, S}+\phi_{k, R}\right)\right)}{\sqrt{E_{k, R} D_{k, R}+D_{k, 0}}} x(k)+\tilde{v}_{D_{2}}(k)$

where $\tilde{v}_{D_{2}}(k)$ is the output noise with variance $N_{0}$. In (6), $D_{k, 0}, D_{k, 1}, D_{k, 2}$, and $D_{k, R}$ are defined as $D_{k, 0}=E G_{S R}\left|D_{S R}(k, k)\right|^{2}+N_{0}, D_{k, 1}=$ $D_{S D}(k, k), D_{k, 2}=\sqrt{G_{S R} G_{R D}} D_{S R}(k, k) D_{R D}(k, k)$ and $D_{k, R}=G_{R D}\left|D_{R D}(k, k)\right|^{2}$. Based on the received signals given by (1) and (6) and assuming perfect CSI, the destination node performs ML detection using the metric

$$
\begin{gathered}
\hat{x}(k)=\underset{x(k)}{\arg \min }\left\{\left|r_{D_{1}}(k)-\sqrt{E_{k, S}} \exp \left(j \phi_{k, S}\right) D_{k, 1} x(k)\right|^{2}\right. \\
\left.+\left|\tilde{r}_{D_{2}}(k)-\frac{\sqrt{E_{k, S} E_{k, R}} \exp \left(j\left(\phi_{k, S}+\phi_{k, R}\right)\right) D_{k, 2}}{\sqrt{E_{k, R} D_{k, R}+D_{k, 0}}} x(k)\right|^{2}\right\}
\end{gathered}
$$

\section{AdAptive LoAding Algorithms for BER OPTIMIZATION}

In this section, we propose three adaptive bit and/or power loading algorithms to minimize the BER. Based on the availability of instantaneous CSI, approximate BER expressions for M-PSK and rectangular M-QAM are given by [23]

$$
P \approx \sum_{k=1}^{N} c_{k} Q\left(\sqrt{a_{k} \varepsilon_{k}}\right)
$$

where $c_{k}, a_{k}$ and $\varepsilon_{k}$ are defined as

$$
\varepsilon_{k}=E_{k, S}\left|D_{k, 1}\right|^{2}+\frac{E_{k, S} E_{k, R}\left|D_{k, 2}\right|^{2}}{\left(E_{k, R} D_{k, R}+D_{k, 0}\right)}
$$

$$
\begin{gathered}
c_{k}= \begin{cases}\frac{\left(M_{k}-1\right)}{\sum_{k=1}^{N} \log _{2} M_{k}}, & \text { for } \mathrm{M}-\mathrm{PSK} \\
\frac{4}{\sum_{k=1}^{N} \log _{2} M_{k}}\left(\frac{\sqrt{M_{k}}-1}{\sqrt{M_{k}}}\right), & \text { for } \mathrm{M}-\mathrm{QAM}\end{cases} \\
a_{k}= \begin{cases}\frac{2}{N_{0}} \sin ^{2}\left(\frac{\pi}{M_{k}}\right), & \text { for } \mathrm{M}-\mathrm{PSK} \\
\frac{3}{N_{0}\left(M_{k}-1\right)}, & \text { for } \mathrm{M}-\mathrm{QAM}\end{cases}
\end{gathered}
$$

where $M_{k}$ is the constellation size for the $k^{\text {th }}$ subcarrier.

\section{A. Optimal Power Loading (OPL)}

In this subsection, we aim to find OPL coefficients for source and relay subcarriers to minimize the BER under total power constraint and fixed subcarrier rate (i.e., fixed modulation scheme for all subcarriers). Total power constraint dictates $(1 / N) \sum_{k=1}^{N}\left(E_{k, S}+E_{k, R}\right)=2 E$. On the other hand, under fixed subcarrier rate, we have $M_{k}=M$ which yields constant values $c_{k}=c$ and $a_{k}=a$ for all subcarriers. Let us define $\mathbf{E}_{k, S}=\left[\begin{array}{llll}E_{1, S} & E_{2, S} & \cdots & E_{N, S}\end{array}\right]^{T}$ and $\mathbf{E}_{k, R}=\left[\begin{array}{llll}E_{1, R} & E_{2, R} & \cdots & E_{N, R}\end{array}\right]^{T}$ as the vectors representing source and relay power loading coefficients. Therefore, the optimization problem can be expressed as

$$
\min _{\mathbf{E}_{k, S}, \mathbf{E}_{k, R}} c \sum_{k=1}^{N} Q\left(\sqrt{a \varepsilon_{k}}\right)
$$


subject to the constraint of

$$
\frac{1}{N} \sum_{k=1}^{N}\left(E_{k, S}+E_{k, R}\right)=2 E, \quad E_{k, S}>0, \quad E_{k, R} \geq 0
$$

Since $Q($.$) is convex, objective function in (12) is also$ convex as proved in the appendix. Therefore, its solution will provide global optimum results.

Factoring the constraint into the objective function, we formulate Lagrangian problem as

$$
\begin{aligned}
\Psi= & c \sum_{k=1}^{N} Q\left(\sqrt{a \varepsilon_{k}}\right)+\lambda\left(\frac{1}{N} \sum_{k=1}^{N}\left(E_{k, S}+E_{k, R}\right)-2 E\right) \\
& -\sum_{k=1}^{N} \mu_{k} E_{k, S}-\sum_{k=1}^{N} \eta_{k} E_{k, R}
\end{aligned}
$$

where $\lambda$ is the Lagrange multiplier. Eq. (13) can be rewritten as

$$
\nabla_{g} \Psi=\mathbf{0}
$$

where we define $\mathbf{g}$ as

$$
\begin{array}{r}
\mathbf{g}=\left[E_{1, S}, \cdots, E_{N, S}, E_{1, R}, \cdots, E_{N, R}, \mu_{1}, \cdots \mu_{N},\right. \\
\left.\eta_{1}, \cdots \eta_{N}, \lambda, \phi_{1, S}, \cdots, \phi_{N, S}, \phi_{1, R}, \cdots, \phi_{N, R}\right]
\end{array}
$$

and $\nabla_{g}$ is the gradient operator with respect to elements of $\mathbf{g}$. Since $\Psi$ is independent of $\phi_{k, S}$ and $\phi_{k, R}$, they will not affect the optimization. Therefore, we set $\phi_{k, S}=\phi_{k, R}=0$. KarushKuhn-Tucker (KKT) conditions for the optimization problem at hand can be then written as [24]

$$
\begin{gathered}
\frac{\partial \Psi}{\partial E_{k, S}}=-c \sqrt{\frac{a}{8 \pi \varepsilon_{k}}} \exp \left(-\frac{a \varepsilon_{k}}{2}\right) \frac{\partial \varepsilon_{k}}{\partial E_{k, S}}+\frac{\lambda}{N}-\mu_{k}=0 \\
\frac{\partial \Psi}{\partial E_{k, R}}=-c \sqrt{\frac{a}{8 \pi \varepsilon_{k}}} \exp \left(-\frac{a \varepsilon_{k}}{2}\right) \frac{\partial \varepsilon_{k}}{\partial E_{k, R}}+\frac{\lambda}{N}-\eta_{k}=0 \\
\frac{1}{N} \sum_{k=1}^{N}\left(E_{k, S}+E_{k, R}\right)-2 E=0 \\
\mu_{k} E_{k, S}=0 \\
\eta_{k} E_{k, R}=0 \\
E_{k, R} \geq 0, \quad E_{k, S}>0 \\
\eta_{k} \geq 0, \quad \mu_{k} \geq 0, \lambda \geq 0
\end{gathered}
$$

Optimum values of power loading coefficients $E_{k, S}, E_{k, R}$ can be then obtained by simultaneously solving (17)-(23). Eqs. (22) and (23) have the following four possible solutions:

$$
\begin{gathered}
\mu_{k}=0, \quad \eta_{k}=0 \\
\mu_{k}=0, \quad E_{k, R}=0 \\
\eta_{k}=0, \quad E_{k, S}=0 \\
E_{k, S}=0, \quad E_{k, R}=0
\end{gathered}
$$

The third and fourth solutions in (26) and (27) are not feasible and can be ignored under our assumption of fixed subcarrier rate. The second solution (25) means no cooperation for the $k^{\text {th }}$ subcarrier and is adopted only for unreliable subcarriers (which will be elaborated later). On the other hand, solution (24) represents the cooperation case which is adopted for reliable subcarriers. Inserting $\mu_{k}=0, \eta_{k}=0$ in (17) and (18), we can readily find out $\partial \varepsilon_{k} / \partial E_{k, S}=\partial \varepsilon_{k} / \partial E_{k, R}$. Using this relation and after some mathematical mainpulations, we can express $E_{k, S}$ in terms of $E_{k, R}$ as

$$
\begin{aligned}
E_{k, S}= & \frac{D_{k, R}}{D_{k, 0}}\left(\frac{\left|D_{k, 1}\right|^{2} D_{k, R}}{\left|D_{k, 2}\right|^{2}}+1\right) E_{k, R}^{2} \\
& +\left(2 D_{k, R} \frac{\left|D_{k, 1}\right|^{2}}{\left|D_{k, 2}\right|^{2}}+1\right) E_{k, R}+\frac{D_{k, 0}\left|D_{k, 1}\right|^{2}}{\left|D_{k, 2}\right|^{2}}
\end{aligned}
$$

then we can rewrite (14) in terms of $E_{k, R}$ as

$$
\begin{aligned}
\Psi= & c \sum_{k=1}^{N} Q\left(\sqrt{a \varepsilon_{k}}\right) \\
& +\lambda\left(\frac{1}{N} \sum_{k=0}^{N}\left(\frac{D_{k, R}}{D_{k, 0}} \gamma_{k} E_{k, R}^{2}+2 \gamma_{k} E_{k, R}+\alpha_{k}\right)-2 E\right)
\end{aligned}
$$

where $\varepsilon_{k}$ is expressed in terms of $E_{k, R}$ as

$$
\varepsilon_{k}=\frac{\left|D_{k, 2}\right|^{2}}{D_{k, 0}}\left(\gamma_{k} E_{k, R}+\alpha_{k}\right)^{2}
$$

Here, $\alpha_{k}$ and $\gamma_{k}$ are defined as

$$
\begin{gathered}
\alpha_{k}=D_{k, 0}\left|D_{k, 1}\right|^{2} /\left|D_{k, 2}\right|^{2} \\
\gamma_{k}=1+D_{k, R}\left|D_{k, 1}\right|^{2} /\left|D_{k, 2}\right|^{2}
\end{gathered}
$$

Solving $\partial \Psi / \partial E_{k, R}=0$ yields

$$
\begin{aligned}
f\left(E_{k, R}\right)= & \exp \left(-a \frac{\left|D_{k, 2}\right|^{2}}{2 D_{k, 0}}\left(\gamma_{k} E_{k, R}+\alpha_{k}\right)^{2}\right) \\
& -\lambda\left(\frac{\sqrt{8 \pi}\left(D_{k, R} E_{k, R}+D_{k, 0}\right)}{c N\left|D_{k, 2}\right| \sqrt{a D_{k, 0}}}\right)=0
\end{aligned}
$$

From (33), it can be found out that some relay subcarriers will have negative power values if the following condition given as

$$
\exp \left(-a \frac{\left|D_{k, 2}\right|^{2}}{2 D_{k, 0}} \alpha_{k}^{2}\right)<\lambda\left(\frac{\sqrt{8 \pi D_{k, 0}}}{c N\left|D_{k, 2}\right| \sqrt{a}}\right)
$$

is satisfied. These basically correspond to unreliable relay subchannels. Since negative power is physically meaningless, the power of such subcarriers should be forced to zero. This indicates that the optimum solution will yield the noncooperative case for the unreliable subcarriers. Replacing $\mu_{k}=0, \quad E_{k, R}=0$ in (17) and (18) and imposing KKT conditions, we have

$$
E_{k, S}=\frac{1}{a\left|D_{k, 1}\right|^{2}} W\left(\left(\frac{\left|D_{k, 1}\right|^{2} c a N}{2 \sqrt{2 \pi} \lambda}\right)^{2}\right)
$$

where $W$ in (35) is the Lambert function, i.e., the inverse function of $f(x)=x \exp (x)$ [25]. Based on the above derivation steps, we can summarize the proposed algorithm as in Algorithm 1.

As an example, we illustrate power distribution among subcarriers for given CSIs in Fig. 3. The curves labelled by 

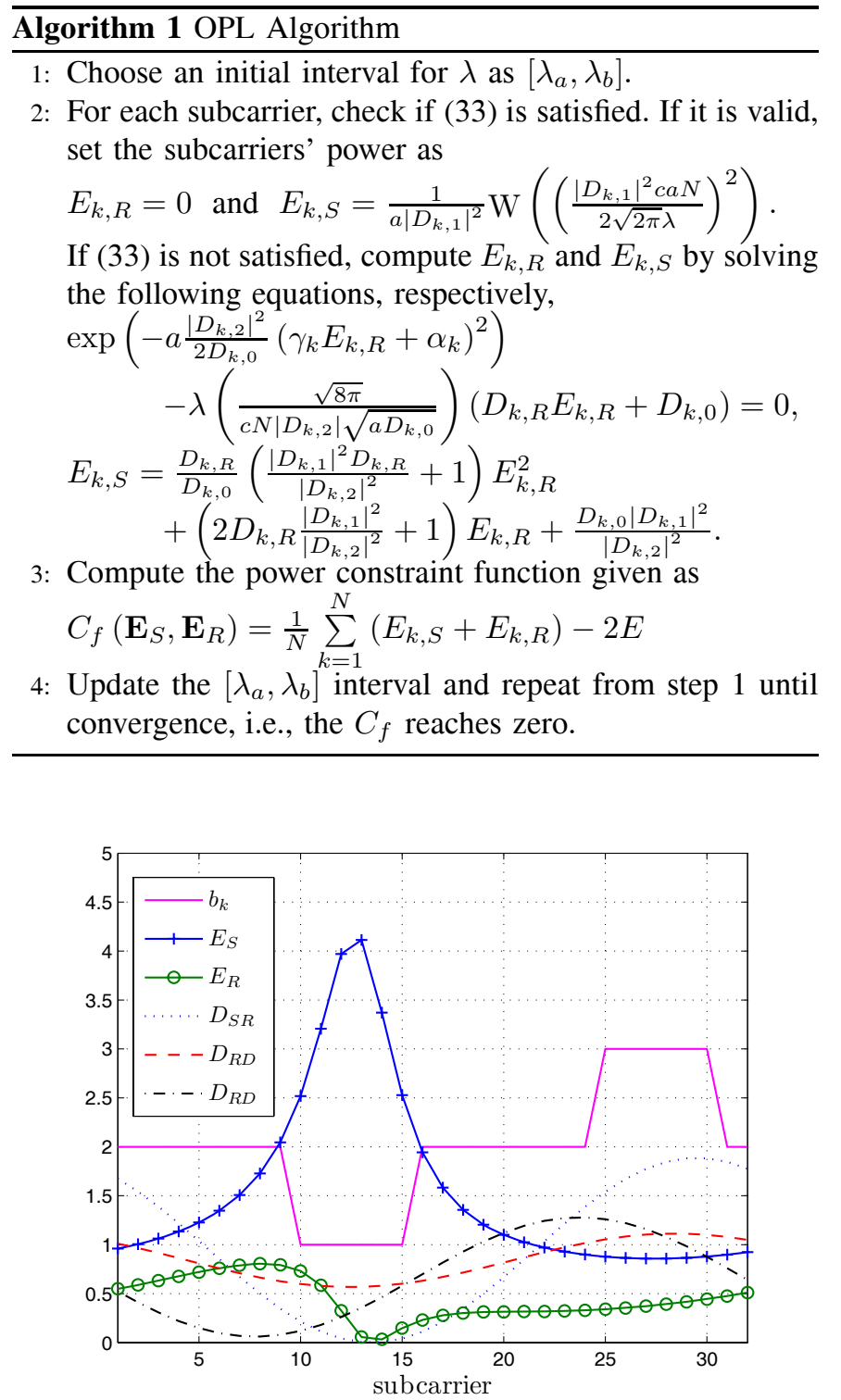

Fig. 3. Power and loading coefficients for a given channel realization.

$D_{S D}, D_{S R}$, and $D_{R D}$ denote fading channel realizations. The curves labelled by $E_{S}$ and $E_{R}$ denote the power loading coefficients for source and relay assuming $N=32$. It can be observed that OPL algorithm effectively equalizes bad subchannels assigning more power onto them.

\section{B. Optimal Bit Loading $(O B L)$}

In this subsection, we aim to find OBL coefficients assuming equal power loading and fixed average transmission rate. Under these assumptions, we have $E_{k, S}=E_{k, R}=E \quad \forall k$ and $\sum_{k=1}^{N} b_{k}=N B$ where $b_{k}=\log _{2} M_{k}$ denotes the bit value assigned to the $k^{\text {th }}$ subcarrier bit loading coefficient. Therefore, the optimization problem is given by

$$
\min _{\mathbf{b}} \sum_{k=1}^{N} c_{k} Q\left(\sqrt{a_{k} \varepsilon_{k}}\right)
$$

subject to the following constraints

$$
\sum_{k=1}^{N} b_{k}=N B, \quad 0 \leq b_{k} \leq N B, \quad E_{k, S}=E_{k, R}=E \quad \forall k
$$

The optimization problem of finding $\mathbf{b}=\left[b_{1}, b_{2}, \ldots b_{N}\right]$ is classified as an integer optimization problem of separable objective functions. The optimal solution can be found using the dynamic programming approach [26] where the objective function in (36) is simplified by dividing it into simpler subproblems which are then solved recursively. In our case, we define the recursive functions associated with sub-problems as

$$
f^{(k)}(p)=\min _{\mathbf{b}_{j}} \sum_{j=1}^{k} f_{j}\left(b_{j}\right)
$$

subject to $\sum_{j=1}^{k} b_{j}=p$. Here, $f_{j}\left(b_{j}\right)=c_{j} Q\left(\sqrt{a_{j} \varepsilon_{j}}\right), b_{j}$ is a non-negative integer $j=1,2, \cdots, k, k=1,2, \cdots, N$ and $p=0,1, \cdots, N B$. Starting with the initial condition $f^{(1)}(p)=f_{1}(p), p=0,1, \cdots, N B$ we recursively need to compute $f^{(k)}(p)$ which are functions of $c_{p}$ and $a_{p}$, c.f. (10) and (11). At $k=N$ and $p=N B$, we obtain $f^{(N)}(N B)$ which yields the OBL coefficients. The proposed algorithm can be summarized as in Algorithm 2. As an example, we illustrate bit loading values for given CSIs in Fig. 3. As expected, OBL algorithm loads more bits to reliable channels.

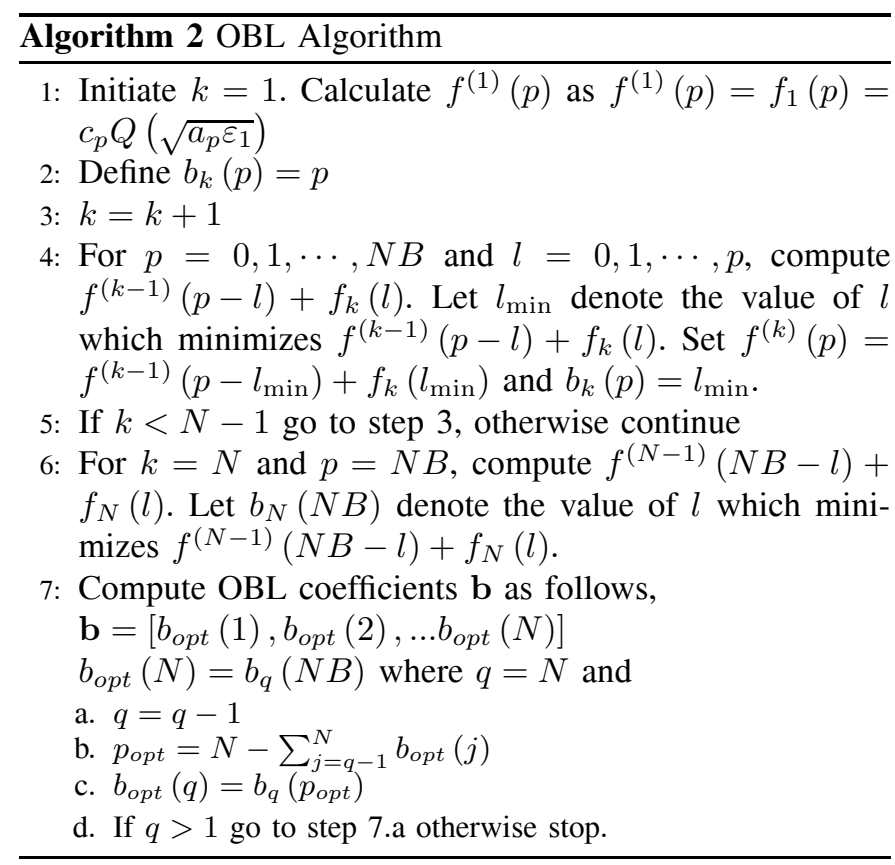

\section{Optimal Bit and Power Loading (OBPL)}

In the previous two problems, we have fixed either subcarrier power or subcarrier rate and computed the optimal value for the other parameter. In this section, we present a joint OBPL scheme which simultaneously optimizes subcarrier rate and power to minimize BER. This problem can be expressed 
as

$$
\min _{\mathbf{E}_{S}, \mathbf{E}_{R}, \mathbf{b}} \gamma\left(\mathbf{E}_{S}, \mathbf{E}_{R}, \mathbf{b}\right)=\sum_{k=1}^{N} c_{k} Q\left(\sqrt{a_{k} \varepsilon_{k}}\right)
$$

subject to the following constraints

$$
\begin{aligned}
& \frac{1}{N} \sum_{k=1}^{N}\left(E_{k, S}+E_{k, R}\right)=2 E, \quad E_{k, S}>0, \quad E_{k, R} \geq 0, \\
& \sum_{k=1}^{N} b_{k}=B, \quad 0 \leq b_{k} \leq N B
\end{aligned}
$$

The current problem is a mixed integer nonlinear convex optimization problem [27]. Here, we use Generalized Benders Decomposition (GBD) method [27] which converts the joint optimization problem into upper and lower bound optimization problems. The upper bound problem is a nonlinear optimization problem for the computation of the power loading coefficients. On the other hand, the lower bound problem is an integer optimization problem for the computation of the bit loading coefficients. The joint optimal solution can be obtained by iteratively solving the two problems each of which uses the others output until convergence. The proposed OPBL algorithm based on GBD method can be summarized as in Algorithm 3.

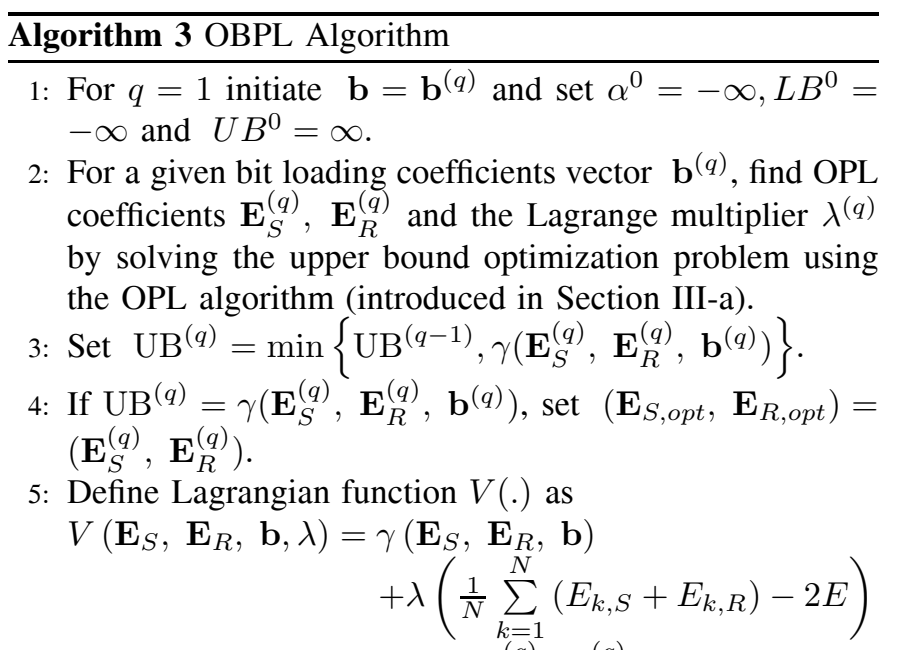
Using the OPL coefficients $\left(\mathbf{E}_{S}^{(q)}, \mathbf{E}_{R}^{(q)}\right)$ obtained in step 2, update the bit loading coefficients $b$ through solving the lower bound integer optimization problem

$$
\begin{aligned}
\min _{\mathbf{b}^{(q+1)}} & V\left(\mathbf{E}_{S}^{(q)}, \mathbf{E}_{R}^{(q)}, \mathbf{b}^{(q)}, \lambda^{(q)}\right) \\
& +\nabla_{\mathbf{M}}^{T} V\left(\mathbf{E}_{S}^{(q)}, \mathbf{E}_{R}^{(q)}, \mathbf{b}^{(q)}, \lambda^{(q)}\right)\left(2^{\mathbf{b}}-2^{\mathbf{b}^{(q)}}\right)
\end{aligned}
$$$$
\text { subject to } \sum_{k=1}^{N} b_{k}^{(q)}=N B \text { and } \alpha^{(q)} \geq \alpha^{(q-1)} \text {, where }
$$$$
\begin{aligned}
\alpha^{(q)} & =V\left(\mathbf{E}_{S}^{(q)}, \mathbf{E}_{R}^{(q)}, \mathbf{b}^{(q)}, \lambda^{(q)}\right) \\
& +\nabla_{\mathbf{M}}^{T} V\left(\mathbf{E}_{S}^{(q)}, \mathbf{E}_{R}^{(q)}, \mathbf{b}^{(q)}, \lambda^{(q)}\right)\left(2^{\mathbf{b}^{(q+1)}}-2^{\mathbf{b}^{(q)}}\right) .
\end{aligned}
$$

(see Algorithm 4 for performing step (5))

6: Set $L B^{(q)}=\alpha^{(q)}$

7: If $L B^{(q)} \geq U B^{(q)}$, stop and the optimal solution will be given as $\left(\mathbf{E}_{S, o p t}, \mathbf{E}_{R, o p t}, \mathbf{b}_{o p t}\right)=\left(\mathbf{E}_{S}^{(q)}, \mathbf{E}_{R}^{(q)}, \mathbf{b}^{(q)}\right)$. Otherwise, set $q=q+1$ and go to step 2 .

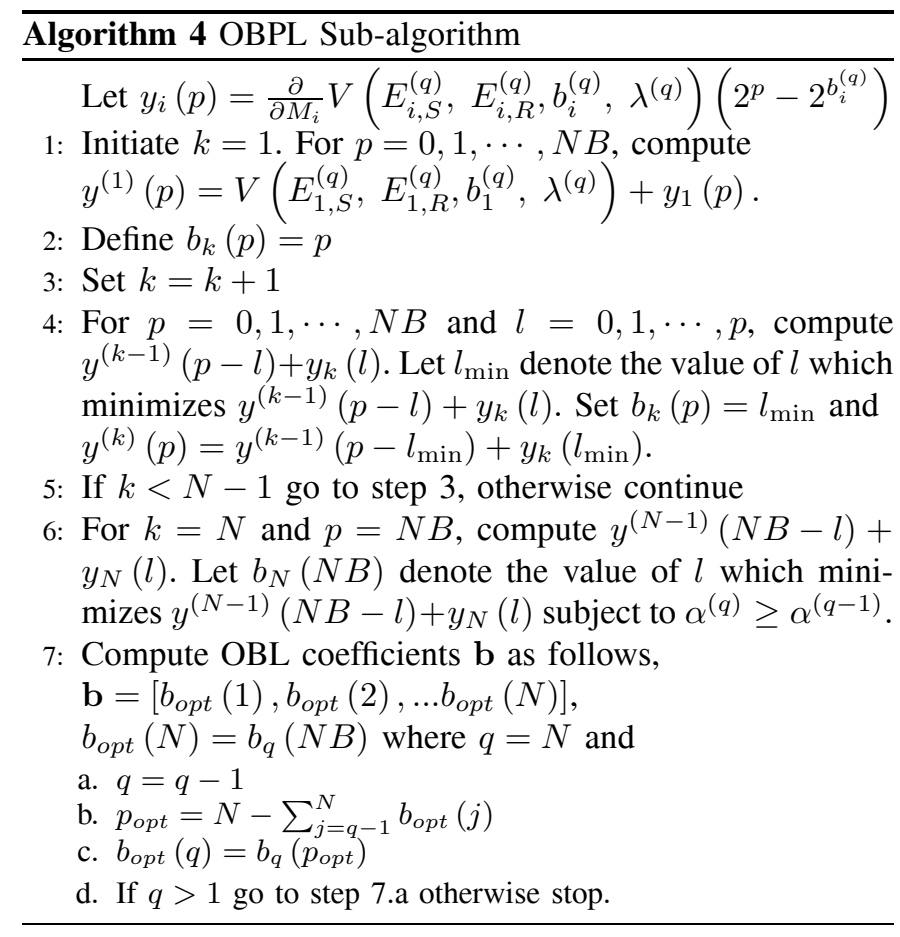

\section{Simulation Results}

In this section, we investigate the BER performance of proposed OPL, OBL, and OBPL algorithms through Monte Carlo simulations. We assume $\alpha=2, \theta=\pi$ and $N=32$. The channel lengths for the three links are assumed to be equal to 2, i.e., $L_{S R}=L_{R D}=L_{S D}=L=1$, SNR is defined to be $E / N_{0}$, where $E$ is the average subcarrier power.

\section{Example 1 (Performance of OBPL)}

In this simulation example, we study the performance of the OBPL algorithm with perfect CSI assuming an average transmission rate of 2 and 4 bits/subcarrier, respectively, in Figs 4 and 5 . We consider $G_{S R} / G_{R D}=30 \mathrm{~dB},-30 \mathrm{~dB}$, and $0 \mathrm{~dB}$. These respectively correspond to cases where the relay is close to the source, close to the destination, and at the midpoint between the source and the destination. The performance of non-adaptive scheme with equal bit and power loading (EBPL) is included as a benchmark. For comparative observations on the diversity order, the performances of co-located multiantenna single-input single-output (SISO) systems with 2, 3 and 4 antennas assuming maximal ratio combining (MRC) are included as further benchmarks.

As observed in Figs. 4 and 5, EBPL scheme achieves only a diversity of two. This diversity gain results from the spatial diversity for the single-relay scenario under consideration. Therefore, EBPL system is not able to extract the underlying multipath diversity. On the other hand, the proposed OBPL scheme is able to extract a diversity order of $2(L+1)=4$ and significantly outperforms EBPL scheme. For example, in Fig. 2, at $\mathrm{BER}=10^{-3}$, OBPL outperforms EBPL by $3.5 \mathrm{~dB}$ assuming $G_{S R} / G_{R D}=0 \mathrm{~dB}$. This climbs up to $5.8 \mathrm{~dB}$ for $G_{S R} / G_{R D}=-30 \mathrm{~dB}$. It is also interesting to note that each scheme attains its best performance at different locations. EBPL attains its best performance when the relay is at the midpoint (i.e., $G_{S R} / G_{R D}=0 \mathrm{~dB}$ ). On the other hand, the performance of OBPL gets better when the relay is placed 


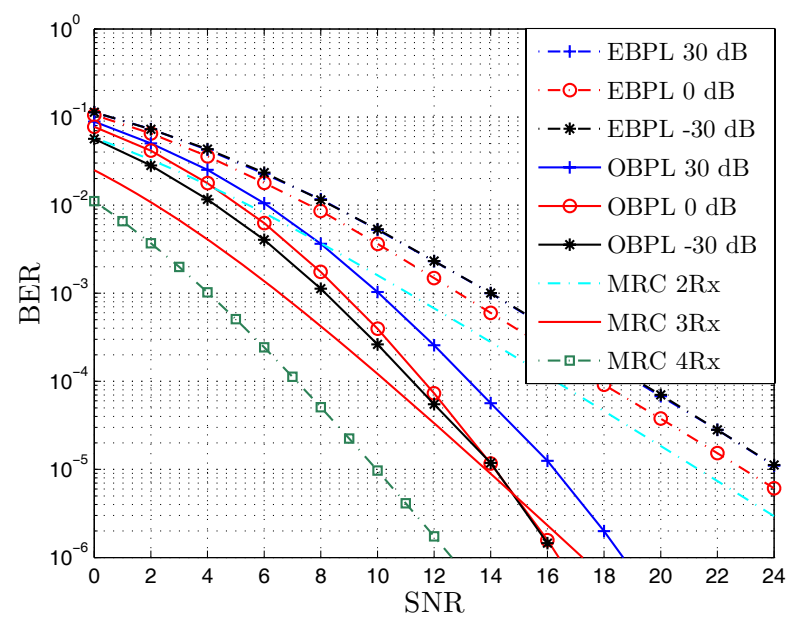

Fig. 4. Performance comparison of EBPL and OBPL schemes for different relay locations with average transmission of $2 \mathrm{bits} / \mathrm{subcarrier}$.

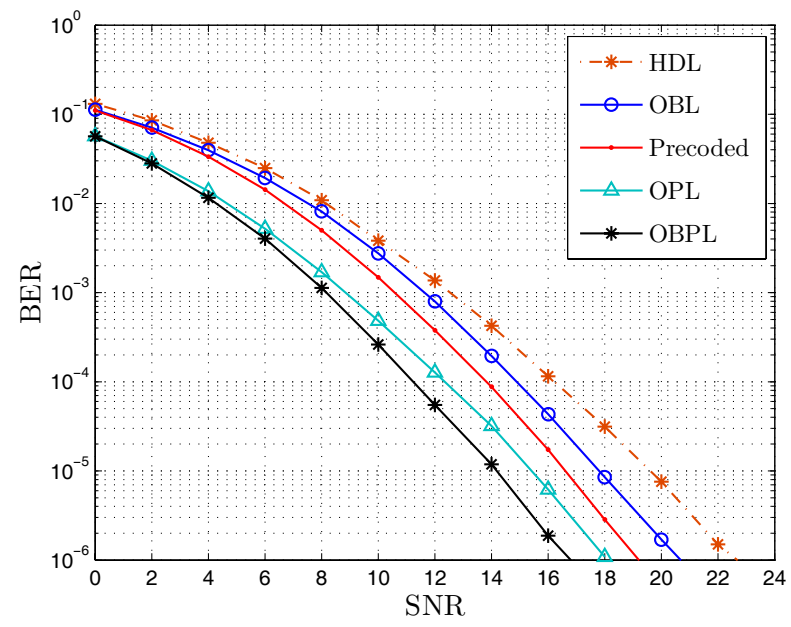

Fig. 5. Performance comparison of EBPL and OBPL schemes for different relay locations with average transmission of $4 \mathrm{bits} /$ subcarrier.

close to the destination (i.e., $G_{S R} / G_{R D}=-30 \mathrm{~dB}$ ). Similar observations hold for Fig. 3 where an average transmission rate of 4 bits/subcarrier is assumed, where at BER $=10^{-3}$, OBPL outperforms EBPL by $3 \mathrm{~dB}$ assuming $G_{S R} / G_{R D}=0 \mathrm{~dB}$. Also the improvement reaches its maximum at $G_{S R} / G_{R D}=$ $-30 \mathrm{~dB}$ where $5 \mathrm{~dB}$ is achieved. From these results, we conclude that dealing with higher rates reduces performance improvement.

\section{Example 2 (OPBL versus other Schemes)}

In this example, we first compare the performance of OBPL to OPL and OBL schemes. Through this comparison, we are particularly interested in finding out whether bit or power loading is more rewarding in performance optimization. In our simulations, we assume perfect CSI, an average transmission rate of 2 bits/subcarrier and $G_{S R} / G_{R D}=-30 \mathrm{~dB}$. From Fig. 6 , we observe that, at $\mathrm{BER}=10^{-4}$, performance gap between OBPL and OBL is $3.6 \mathrm{~dB}$. On the other hand, performance gap between OBPL and OPL reduces to $1.1 \mathrm{~dB}$. Therefore, it can be concluded that power loading is more dominant in performance optimization.

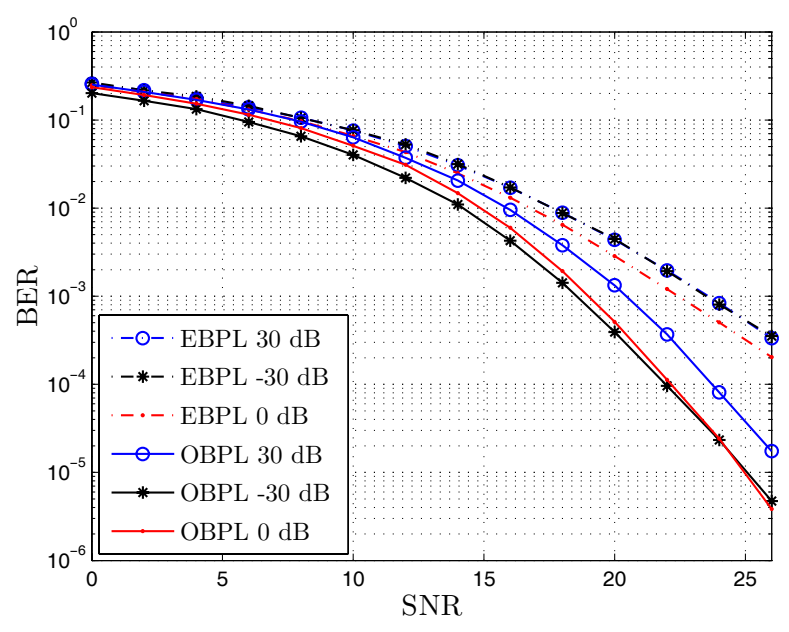

Fig. 6. Performance comparison of OBPL, OPL, OBL algorithms and competing schemes.

For comparison with existing systems in the literature, we also include the performance of a precoded cooperative OFDM [28] in Fig. 6. It is observed that the proposed schemes and the precoded system are both able to extract the full diversity and achieve the same diversity order. However, OBPL and OPL systems are able to outperform the precoded system by $2.6 \mathrm{~dB}$ and $1.5 \mathrm{~dB}$, respectively, at $\mathrm{BER}=10^{-4}$. On the other hand, OBL remains inferior to the precoded system by $\sim 1 \mathrm{~dB}$. It should be further emphasized that, besides performance improvements, OBPL and OPL have advantage over the precoded systems in terms of receiver complexity. The receiver complexity of proposed algorithms is independent of the channel length while the detector complexity in precoded systems is exponentially proportional to the channel length [28].

Another comparison in Fig. 6 is with the power loading scheme proposed in [20] by Hajiaghayi et.al. which also aims to optimize the BER performance (named as HDL scheme in our figure). Our results illustrate the superiority of proposed algorithms over HDL scheme. Specifically at BER $=10^{-3}$, we observe that OBL, OPL and OBPL outperform HDL by 0.8 $\mathrm{dB}, 3.2 \mathrm{~dB}$ and $4.3 \mathrm{~dB}$ respectively. It should be noted that HDL scheme is derived under the high SNR assumption and considers identical subcarrier power at source and relay nodes. Our schemes avoid such restricting assumptions and are therefore able to provide a better performance.

\section{Example 3 (Effect of Relay Location)}

In this example, we study the effect of relay location on the performance of EBPL, OBL, OPL, and OBPL schemes. Under the assumption of average transmission rate of 2 bits/subcarrier and a target fixed BER of $10^{-3}$, we plot the required SNR versus the relay location. Fig. 7 illustrates that the best location (i.e. requires the lowest SNR to achieve the BER $=10^{-3}$ ) for OBPL and OPL is near to destination. For OBL and EBPL, mid-locations between source and destination become more favourable. For near-to-destination and near-to-source locations, they exhibit identical channel statistical properties [29] and therefore yield symmetric performance around $0 \mathrm{~dB}$ location. 


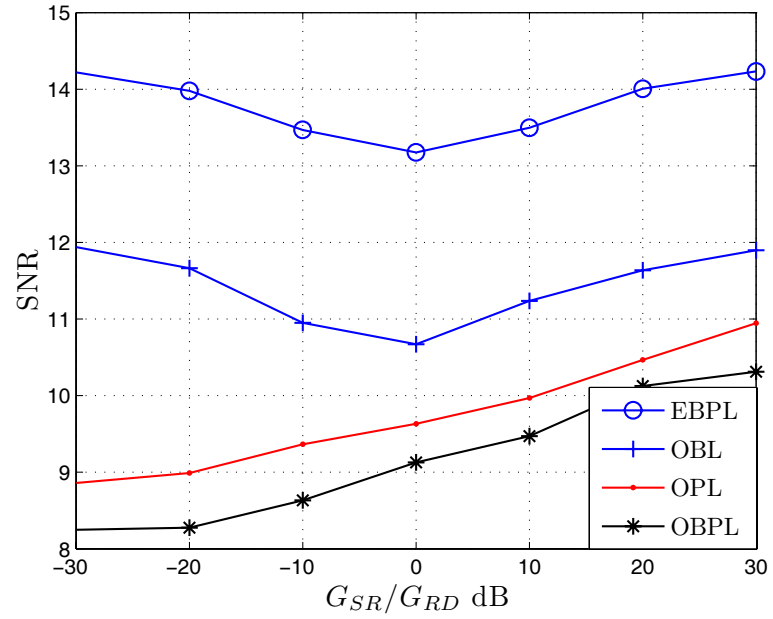

Fig. 7. Effect of relay location on different adaptive algorithms.

\section{Example 4 (Effect of Channel Estimation)}

In this example, we study the effect of channel estimation on the proposed schemes. The adaptive algorithms assume that perfect CSI is available at source, relay and destination nodes. In practice, CSI information needs to be estimated. CSI for the direct link (i.e., $\mathrm{S} \rightarrow \mathrm{D}$ ) and relaying link (i.e., $\mathrm{S} \rightarrow \mathrm{R}$ and $\mathrm{R} \rightarrow \mathrm{D})$ is also used at the destination for detection process. For the estimation of relaying path, we adopt the socalled disintegrated channel estimation (D-CE) approach [29] in which $\mathrm{S} \rightarrow \mathrm{R}$ and $\mathrm{R} \rightarrow \mathrm{D}$ channels are estimated separately. In this approach, the relay node is equipped with a channel estimator and feed-forwards the $\mathrm{S} \rightarrow \mathrm{R}$ channel estimate to the destination terminal as well as feedbacks it to the source. Channel estimates for $\mathrm{S} \rightarrow \mathrm{D}$ and $\mathrm{R} \rightarrow \mathrm{D}$ links are obtained at the destination which sends them to the source and the relay via a feedback channel.

In Fig. 8, we assume the employment of linear minimum mean squared error estimator (LMMSE) [30] and perfect feedback of the estimates. We consider the case where the relay is near to the destination, i.e., $G_{S R} / G_{R D}=-30 \mathrm{~dB}$ and an average transmission rate of 2 bits/subcarrier. Note that the precoded system which is used as a benchmark does not need CSI at the transmitter side. Fig. 8 illustrates that, at $\mathrm{BER}=10^{-4}$, OBPL and OPL schemes with imperfect channel estimation at the transmitter side are still able to outperform the precoded system by $2.3 \mathrm{~dB}$ and $0.9 \mathrm{~dB}$.

In Fig. 9, we study the effect of finite-rate feedback which is required to transfer the quantized CSIs in the practical implementation of our proposed schemes. Fig. 9 shows that we need as small as 5 bits to achieve a similar performance to the ideal system with perfect feedback. When 6 bits is used, it gives an identical performance to that of perfect feedback. The corresponding performance curve is not included in the figure for the sake of presentation.

\section{CONCLUSION}

In this paper, we have investigated adaptive bit and/or power loading for a cooperative OFDM system with AF relaying. We have adopted BER as the objective function and

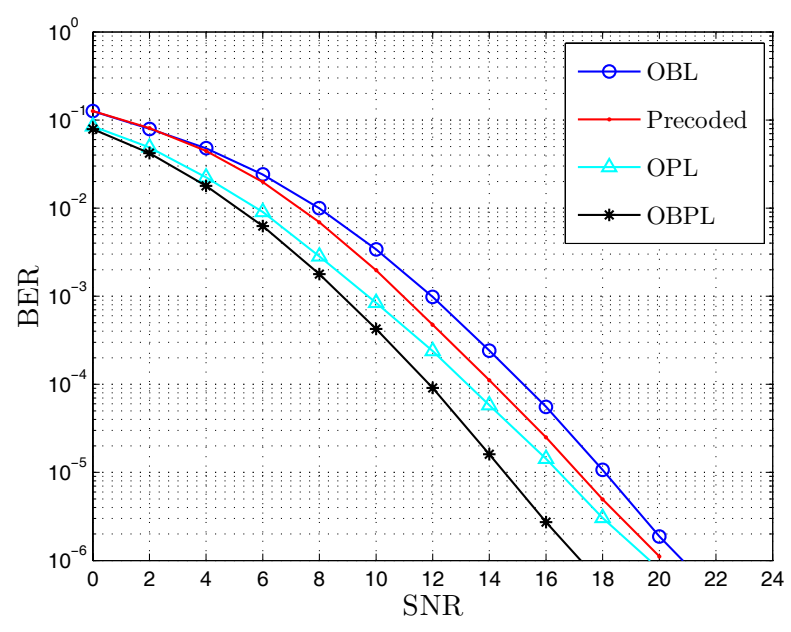

Fig. 8. Performance of OBPL, OPL, OBL algorithms with imperfect channel estimation.

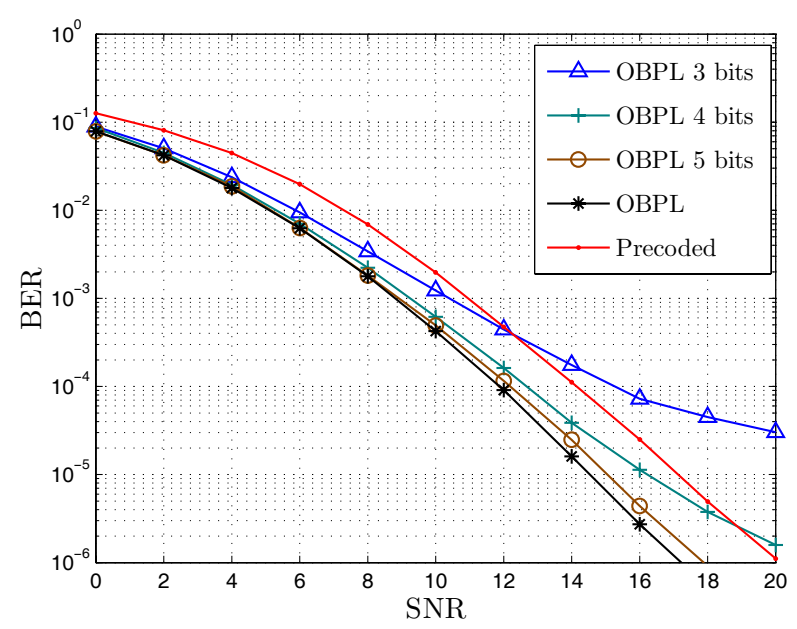

Fig. 9. Effect of finite-rate feedback on the performance of OPBL algorithm.

formulated three optimization problems leading to different adaptive algorithms. The first algorithm, named as OPL, computes the optimal source and relay power loading coefficients under total power constraint and fixed subcarriers rate. The second algorithm, named as OBL, computes the optimal bit loading coefficients under fixed average transmission rate and equal power loading. The third one, named as OBPL, computes the joint optimal power and bit loading coefficients. Through Monte-Carlo simulations, we have demonstrated the superiority of our schemes over conventional non-adaptive cooperative OFDM systems. For example, assuming relay is located close to the destination, OBPL outperforms nonadaptive system (i.e., equal bit and power loading) by $5.8 \mathrm{~dB}$ at a target BER $=10^{-3}$. OBPL and OPL systems are also able to outperform the precoded cooperative OFDM systems while OBL turns out to be inferior. This also indicates that power loading is more dominant in the performance optimization where continuous optimization gives more degrees of freedom than the discrete optimization deployed in bit loading. We have further provided simulation results to quantify the effect of relay location, imperfect channel estimation and finite- 
rate quantized feedback on the BER performance of proposed schemes.

\section{APPENDIX}

In this appendix, we provide the convexity proofs for OPL, OBL and OPBL optimization problems under consideration.

\section{$O P L$}

For this problem, the general objective function given by (8) reduces to

$$
P \approx c \sum_{k=1}^{N} Q\left(\sqrt{a \varepsilon_{k}}\right) .
$$

Let $f_{1}=Q\left(\sqrt{a \varepsilon_{k}}\right)$. Since $a$ and $\varepsilon_{k}$ are positive quantities, $f_{1}$ is a convex function. We first need to prove that $\varepsilon_{k}$ is concave in $E_{k, S}$ and $E_{k, R}$. This will prove the convexity of $f_{1}$ with respect to $E_{k, S}$ and $E_{k, S}$ [31]. Rewriting $\varepsilon_{k}$ given by (9) as

$$
\varepsilon_{k}=g_{1}\left(E_{k, S}, E_{k, R}\right)+g_{2}\left(E_{k, S}, E_{k, R}\right)
$$

where $g_{1}\left(E_{k, S}, E_{k, R}\right)=E_{k, S}\left|D_{k, 1}\right|^{2}$ and

$$
g_{2}\left(E_{k, S}, E_{k, R}\right)=\left\{\begin{array}{c}
\frac{E_{k, S} E_{k, R}\left|D_{k, 2}\right|^{2}}{\left(E_{k, R} D_{k, R}+D_{k, 0}\right)} \\
\frac{E_{k, S} E_{k, R}\left|D_{k, 2}\right|^{2}}{\left(E_{k, R} D_{k, R}+E_{k, S} G_{S R}\left|D_{S R}(k, k)\right|^{2}+N_{0}\right)}
\end{array}\right.
$$

In (43), we consider two cases. As earlier discussed, the original IPS scaling term is given by (3). The modified version involves replacing $E_{k, S}$ with the average value $E$ in (3).

Modified IPS: For the modified versiom, $g_{2}\left(E_{k, S}, E_{k, R}\right)$ can be expressed as

$$
g_{2}\left(E_{k, S}, E_{k, R}\right)=\frac{\left(\left|D_{k, 2}\right|^{2} / D_{k, R} D_{k, 0}\right)}{\left(\frac{1}{E_{k, R} E_{k, S} D_{k, R}}+\frac{1}{D_{k, 0} E_{k, S}}\right)}
$$

Since $D_{k, 2}, \quad D_{k, 0}, \quad D_{k, R}$ and $E_{k, S}$ are positive quantities $g_{2}\left(E_{k, S}, E_{k, R}\right)$ is concave because $1 /\left(E_{k, R} E_{k, S} D_{k, R}\right)$ and $1 /\left(D_{k, 0} E_{k, S}\right)$ are convex functions. The Hessian matrix of $\mathbf{H}_{1}$ is expressed as

$$
\mathbf{H}_{1}=\frac{1}{D_{k, R} E_{k, S} E_{k, R}}\left[\begin{array}{cc}
\frac{2}{E_{k, R}^{2}} & \frac{1}{E_{k, S} E_{k, R}} \\
\frac{1}{E_{k, S} E_{k, R}} & \frac{2}{E_{k, S}^{2}}
\end{array}\right]
$$

Since $D_{k, R}$ is positive, therefore $\mathbf{H}_{1}$ is positive definite matrix which proves the convexity of $\left[1 /\left(E_{k, R} E_{k, S} D_{k, R}\right)\right]$ term. Thus $g_{2}\left(E_{k, S}, E_{k, R}\right)$ is a concave function.

Original IPS: For the original case, (43) can be expressed as

$$
g_{2}\left(E_{k, S}, E_{k, R}\right)=\frac{\frac{4\left|D_{k, 2}\right|^{2}}{N_{0}^{2} D_{k, R} G_{S R}\left|D_{S R}(k, k)\right|^{2}}}{\frac{1}{E_{k, R} D_{k, R}+\frac{N_{0}}{2}}+\frac{1}{E_{k, S} G_{S R}\left|D_{S R}(k, k)\right|^{2}+\frac{N_{0}}{2}}}
$$
by

The Hessian matrix $\mathbf{H}_{2}$ of the term in denominator is given

$$
\mathbf{H}_{2}=\left[\begin{array}{cc}
\frac{2 G_{S R}\left|D_{S R}(k, k)\right|^{2}}{\left(E_{k, S} G_{S R}\left|D_{S R}(k, k)\right|^{2}+\frac{N_{0}}{2}\right)^{3}} & 0 \\
0 & \frac{2 D_{k, R}}{\left(E_{k, R} D_{k, R}+\frac{N_{0}}{2}\right)^{3}}
\end{array}\right]
$$

Since $G_{S R}, D_{k, R}, N_{0}$ and $\left|D_{S R}(k, k)\right|$ are positive values, $\mathbf{H}_{2}$ is a positive definite matrix which proves the convexity term of the denominator term in (46). Accordingly $g_{2}\left(E_{k, S}, E_{k, R}\right)$ is a concave function. As a result scaling with $E_{k, S}$ or $E$ does not affect the concavity nature of $g_{2}\left(E_{k, S}, E_{k, R}\right)$. Since $g_{1}\left(E_{k, S}, E_{k, R}\right)$ and $g_{2}\left(E_{k, S}, E_{k, R}\right)$ are concave functions, $\varepsilon_{k}$ is also concave function. As a result, $f_{1}=Q\left(\sqrt{a \varepsilon_{k}}\right)$ is convex. Finally, noting that the sum of convex functions is convex, (41) turns out to be convex.

$O B L$

For this problem, the objective function will follow the general form in (8). By using the values of $a_{k}$ and $c_{k}$ for M-PSK in (10) and (11), we have

$$
P \approx \sum_{k=1}^{N} \frac{\left(2^{b_{k}}-1\right)}{m N} Q\left(\sqrt{\frac{2}{N_{0}} \sin ^{2}\left(\frac{\pi}{2^{b_{k}}}\right) \varepsilon_{k}}\right)
$$

By relaxing the integer variable $b_{k}$ to be continuous [26] and defining

$$
f_{2}=\frac{\left(2^{b_{k}}-1\right)}{m N} Q\left(\sqrt{\frac{2}{N_{0}} \sin ^{2}\left(\frac{\pi}{2^{b_{k}}}\right) \varepsilon_{k}}\right)
$$

then $P$ is convex if $f_{2}$ is convex. To test the convexity of $f_{2}$, we obtain the second derivative with respect to $b_{k}$ (which was omitted here due to space limitations) which can be shown to be positive. Then as a result, $f_{2}$ is convex and accordingly $P$ is convex.

\section{$O B P L$}

Replacing $a_{k}$ and $c_{k}$ in for M-PSK in the general form of the objective function in, we have

$$
P=\sum_{k=1}^{N} \frac{\left(2^{b_{k}}-1\right)}{m N} Q\left(\sqrt{\frac{2}{N_{0}} \sin ^{2}\left(\frac{\pi}{2^{b_{k}}}\right) \varepsilon_{k}\left(E_{k, S}, E_{k, R}\right)}\right)
$$

Define $f_{3}$ as

$$
f_{3}=\frac{\left(2^{b_{k}}-1\right)}{m N} Q\left(\sqrt{\frac{2}{N_{0}} \sin ^{2}\left(\frac{\pi}{2^{b_{k}}}\right) \varepsilon_{k}\left(E_{k, S}, E_{k, R}\right)}\right)
$$

To prove the convexity of $P$, we need to prove that $f_{3}$ is convex. First note that $\sin \left(\pi / 2^{b_{k}}\right)$ can be bounded as $0 \leq \sin \left(\pi / 2^{b_{k}}\right) \leq 1$. Noting $Q$ function's monotonically decreasing property, it is straightforward to show that

$$
\frac{\left(2^{b_{k}}-1\right)}{m N} Q\left(\sqrt{\frac{2}{N_{0}} \varepsilon_{k}\left(E_{k, S}, E_{k, R}\right)}\right) \leq f_{3} \leq \frac{\left(2^{b_{k}}-1\right)}{2 m N}
$$

Further define $f_{4}=\left(2^{b_{k}}-1\right) /(2 m N)$ and $f_{5}=$ $\left(\left(2^{b_{k}}-1\right) / m N\right) Q\left(\sqrt{\left(2 / N_{0}\right) \varepsilon_{k}\left(E_{k, S}, E_{k, R}\right)}\right), f_{5}$ is convex function if $f_{3}$ and $f_{4}$ are convex functions. We know that $f_{4}$ is convex function, thus we need to show that $f_{5}$ is convex. $f_{5}$ can be bounded as $f_{6}<f_{5}<f_{7}$ where $f_{6}$ and $f_{7}$ are defined as $f_{6}=Q\left(\sqrt{\left(2 / N_{0}\right) \varepsilon_{k}\left(E_{k, S}, E_{k, R}\right)}\right)$ and $f_{7}=(1 / 2 m N) \exp \left(b_{k}-\left(1 / N_{0}\right) \varepsilon_{k}\left(E_{k, S}, E_{k, R}\right)\right)$. Noting $\exp ($.$) is a convex non-decreasing function and$ $\left(b_{k}-\left(1 / N_{0}\right) \varepsilon_{k}\left(E_{k, S}, E_{k, R}\right)\right)$ is convex, is convex in $\left(b_{k}, E_{k, S}, E_{k, R}\right)$ [31]. Since $f_{6}$ and $f_{7}$ are convex, $f_{5}$ is convex and accordingly $f_{3}$ is convex given that $P$ of the OBPL is convex in $\left(b_{k}, E_{k, S}, E_{k, R}\right)$. 


\section{REFERENCES}

[1] A. Nosratinia, T. Hunter, and A. Hedayat, "Cooperative communication in wireless networks," IEEE Commun. Mag., vol. 42, no. 10, pp. 74-80, Oct. 2004.

[2] J. Laneman and G. Wornell, "Distributed space-time-coded protocols for exploiting cooperative diversity in wireless networks," IEEE Trans. Inf. Theory, vol. 49, no. 10, pp. 2415-2425, Oct. 2003.

[3] A. Goldsmith and S.-G. Chua, "Variable-rate variable-power MQAM for fading channels," IEEE Trans. Commun., vol. 45, no. 10, pp. 12181230, Oct. 1997.

[4] G. Caire, G. Taricco, and E. Biglieri, "Optimum power control over fading channels," IEEE Trans. Inf. Theory, vol. 45, no. 5, pp. 14681489, July 1999.

[5] S. T. Chung and A. Goldsmith, "Degrees of freedom in adaptive modulation: a unified view," IEEE Trans. Commun., vol. 49, no. 9, pp. 1561-1571, Sep. 2001

[6] L. Lin, R. Yates, and P. Spasojevic, "Adaptive transmission with discrete code rates and power levels," IEEE Trans. Commun., vol. 51, no. 12, pp. 2115-2125, Dec. 2003.

[7] T. Keller and L. Hanzo, "Adaptive multicarrier modulation: a convenient framework for time-frequency processing in wireless communications," Proc. IEEE, vol. 88, no. 5, pp. 611-640, May. 2000.

[8] C. Assimakopoulos and F. Pavlidou, "Transmission systems unified models for adaptive OFDM systems when QAM or PSK modulation is applied," European Trans. Telecommun., vol. 18, no. 7, pp. 777-790, Nov. 2007.

[9] N. Ahmed, M. Khojastepour, A. Sabharwal, and B. Aazhang, "Outage minimization with limited feedback for the fading relay channel," IEEE Trans. Commun., vol. 54, no. 4, pp. 659-669, Mar. 2006.

[10] N. Ahmed and B. Aazhang, "Throughput gains using rate and power control in cooperative relay networks," IEEE Trans. Commun., vol. 55, no. 4, pp. 656-660, Apr. 2007.

[11] Y. Gabbai and S. Bross, "Achievable rates for the discrete memoryless relay channel with partial feedback configurations," IEEE Trans. Inf. Theory, vol. 52, no. 11, pp. 4989-5007, Oct. 2006.

[12] I. Hammerstrom and A. Wittneben, "On the optimal power allocation for nonregenerative OFDM relay links," in Proc. IEEE International Conf. on Communications, vol. 10, 2006, pp. 4463-4468.

[13] _ , "Power allocation schemes for amplify-and-forward MIMOOFDM relay links," IEEE Trans. Wireless Commun., vol. 6, no. 8, pp. 2798-2802, Aug. 2007.

[14] W. Ying, Q. Xin-Chun, W. Tong, and L. Bao-Ling, "Power allocation and subcarrier pairing algorithm for regenerative OFDM relay system," in Proc. IEEE 65th Vehicular Technology Conf., Spring 2007, pp. 27272731.

[15] H. Ochiai, P. Mitran, and V. Tarokh, "Design and analysis of collaborative diversity protocols for wireless sensor networks," in Proc. IEEE 60th Vehicular Technology Conf., vol. 7, 2004, pp. 4645-4649.

[16] B. Gui and L. J. Cimini, "Bit loading algorithms for cooperative OFDM systems," EURASIP J. Wireless Commun. and Netw., vol. 2008, 2007.

[17] N. Yi, Y. Ma, and R. Tafazolli, "Bit and power loading for OFDM with an amplify-and-forward cooperative relay," in Proc. IEEE 19th International Symp. on Personal, Indoor and Mobile Radio Communications, 2008, pp. $1-5$.

[18] — , "Rate-adaptive bit and power loading for OFDM based DF relaying," in Proc. IEEE 67th Vehicular Technology Conf., Spring 2008, pp. $1340-1344$.

[19] Y. Ma, N. Yi, and R. Tafazolli, "Bit and power loading for OFDM-based three-node relaying communications," IEEE Trans. Signal Process., vol. 56, no. 7, pp. 3236-3247, July 2008.

[20] M. Hajiaghayi, M. Dong, and B. Liang, "Using limited feedback in power allocation design for a two-hop relay OFDM system," in Proc. IEEE International Conference on Communications, 2009, pp. 1-6.
[21] H. Mheidat and M. Uysal, "Impact of receive diversity on the performance of amplify-and-forward relaying under APS and IPS power constraints," IEEE Commun. Lett., vol. 10, no. 6, pp. 468-470, June 2006.

[22] J. Laneman, "Cooperative diversity in wireless networks: algorithms and architectures," Ph.D. dissertation, 2002.

[23] J. Proakis and M. Salehi, Digital Communications. McGraw-Hill, 2001.

[24] J. Snyman, Practical Mathematical Optimization: An Introduction to Basic Optimization Theory and Classical and New Gradient-Based Algorithms. Springer Verlag, 2005.

[25] R. M. Corless, G. H. Gonnet, D. E. G. Hare, D. J. Jeffrey, and D. E. Knuth, "On the Lambert W function," Adv. Comp. Math, vol. 5, no. 1, pp. 329-359, 1996.

[26] T. Ibaraki and N. Katoh, Resource Allocation Problems: Algorithmic Approaches. MIT Press, 1988.

[27] D. Li and X. Sun, Nonlinear Integer Programming. Springer, 2006.

[28] Z. Liu, Y. Xin, and G. Giannakis, "Linear constellation precoding for OFDM with maximum multipath diversity and coding gains," IEEE Trans. Commun., vol. 51, no. 3, pp. 416-427, Mar. 2003.

[29] O. Amin, B. Gedik, and M. Uysal, "Channel estimation for amplifyand-forward relaying: cascaded against disintegrated estimators," IET Commun., vol. 4, no. 10, pp. 1207-1216, Oct. 2010.

[30] S. Kay, Fundamentals of Statistical Signal Processing: Estimation Theory. Prentice Hall Inc., 1993.

[31] S. Boyd and L. Vandenberghe, Convex Optimization. Cambridge University Press, 2004.

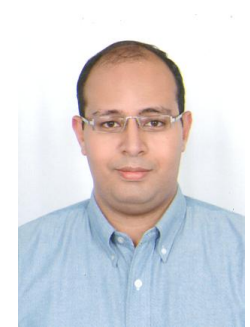

Osama Amin received B.Sc. degree in Electrical and Electronics Engineering from South Valley University, Aswan, Egypt, in 2000 and M.Sc. degree in Electrical Engineering from Assiut University, Assiut, Egypt in 2004. He is currently a Ph.D. student in Electrical and Computer Engineering, University of Waterloo, Canada. His research interests include cooperative communications, adaptive systems, OFDM, and channel estimation.

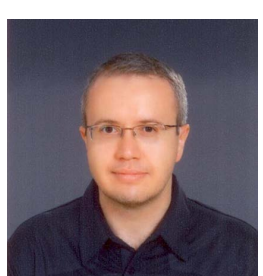

Murat Uysal was born in Istanbul, Turkey in 1973. $\mathrm{He}$ received the B.Sc. and the M.Sc. degree in electronics and communication engineering from Istanbul Technical University, Istanbul, Turkey, in 1995 and 1998, respectively, and the Ph.D. degree in electrical engineering from Texas A\&M University, College Station, Texas, in 2001. Since 2002, he has been with the Department of Electrical and Computer Engineering, University of Waterloo, Canada, where he is now an Associate Professor. He is currently on leave at Özyeğin University, Istanbul, Turkey. His general research interests lie in communications theory and signal processing for communications with special emphasis on wireless applications. Specific research areas include MIMO communication techniques, space-time coding, diversity techniques and coding for fading channels, cooperative communication, and free-space optical communication.

Dr. Uysal is an Associate Editor for IEEE TRANS ACTIONS ON WIRELESS COMMUNICATIONS and IEEE COMMUNiCATIONS LETTERS. He was a Guest Co-Editor for the Wiley Journal on Wireless Communications and Mobile Computing Special Issue on "MIMO Communications" (October 2004), and IEEE Journal on SELECTED AREAS IN COMMUNiCATIONS Special Issue on "Optical Wireless Communications" (December 2009). Over the years, he has served on the technical program committee of more than 60 international conferences in the communications area. He recently cochaired IEEE ICC'07 Communication Theory Symposium and CCECE'08 Communications and Networking Symposium. Dr. Uysal is a Senior IEEE member. 